\title{
Cuidados paliativos una herramienta para estudiantes de enfermería y garantía de la dignidad humana
}

\author{
María Magdalena Alvarado Acevedo ${ }^{1}$ \\ Evelyn Yesenia Cruz Palacios ${ }^{2}$ \\ Estela de Jesús Alvarado Guardado ${ }^{3}$ \\ Docentes investigadoras, Facultad Multidisciplinaria de Ilobasco \\ Universidad Católica de El Salvador, El Salvador
}

Fecha de recepción: $22-12-2020$

Fecha de aceptación: 23-02-2021

\section{Resumen}

La enfermería tiene su razón de ser en el cuidado a la persona y al realizar cuidados paliativos se necesita de profesionales con conocimientos especializados y un alto grado de calidez humana que le permita dar un soporte al paciente y familia en la etapa terminal de la enfermedad. Desde este enfoque, se puede determinar el impacto que tiene el conocimiento especializado que se debe adquirir desde la formación de los estudiantes de enfermería, quienes se convierten en profesionales que prestan sus servicios en diferentes instituciones del sector salud público o privado.

Se investigó, desde el contexto salvadoreño, el conocimiento sobre cuidados paliativos de los estudiantes de $5^{\circ}$ año de Licenciatura en Enfermería de la Universidad Católica de El Salvador, limitando la muestra al Centro Regional de Ilobasco, considerando que es el único centro educativo de nivel superior de la zona paracentral donde se forman profesionales en enfermería íntimamente relacionada a los cuidados directos del paciente.

El propósito del estudio fue identificar conocimientos en estudiantes de enfermería sobre el tipo de pacientes que requieren cuidados paliativos. En consecuencia, la investigación es un precedente importante para realizar esfuerzos para justificar una reforma a la malla curricular de la carrera de licenciatura en enfermería e incorporar como asignatura del área. especializada de enfermería, en la cual, se aborde el contenido de cuidados paliativos de forma holística, humanitaria y que promueva una atención digna en la etapa final de la vida.

Palabras clave: Cuidados paliativos, calidad de vida hasta la muerte, muerte con dignidad, alivio del dolor, cuidado de enfermería, gestión de cuidado paliativo.

\begin{abstract}
Nursing has its reason for being in caring for the person and when performing palliative care, professionals with specialized knowledge and a high degree of human warmth are needed to support the patient and family in the terminal stage of the disease. From this approach, the impact of the specialized knowledge that must be acquired from the training of nursing students, who become professionals providing their services in different institutions of the public or private health sector, can be determined.

Therefore, from the Salvadoran context, the knowledge about palliative care of fifth-year students of the Nursing Degree of Universidad Católica de El Salvador was investigated, limiting the sample to the Regional Center of Ilobasco, considering that it is the only higher level educational center of the paracentral area where nursing professionals closely related to the direct care of the patient are trained.

The purpose of the study was to identify knowledge in nursing students about the type of patients that require palliative care. Consequently, the research is an important precedent to make efforts to justify a reform of the curriculum of the undergraduate degree in nursing and incorporate this area as a subject of the same.

Key words: Palliative care, quality of life until death, death with dignity, pain relief, nursing care, palliative care management.

1. Maestra en asesoría educativa; email: maria.alvarado5@catolica.edu.sv; ORCID: https://orcid.org/0000-0002-8666-1410

2. Maestra en asesoría educativa; email: evelin.cruz@catolica.edu.sv; ORCID: https://orcid.org/0000-0002-8666-1410

3. Maestra en asesoría educativa; email: estela.alvarado5@catolica.edu.sv; ORCID: https://orcid.org/0000-0003-0760-5515
\end{abstract}




\section{Introducción}

La Organización Mundial de la Salud (OMS, 2018) en su definición de cuidados paliativos se refiere a los mismos como un enfoque que mejora la calidad de vida de los pacientes y familiares que se están enfrentando a una enfermedad potencialmente mortal, a través de la prevención y el alivio del sufrimiento realizando una identificación temprana, una evaluación adecuada y aplicando tratamientos para el dolor y otros problemas físicos, psicosociales y espirituales, enfocándose en la última etapa de la enfermedad, en el papel que juega el paciente en esta etapa y en la garantía de los derechos de este aun cuando pudiere pensarse que ha dejado de ser sujeto de derechos.

La razón es que millones de personas sufren enfermedades terminales alrededor del mundo: cuando la muerte es inevitable, la forma de morir es importante porque un convaleciente no es menos digno que los demás. Por ello es notable el hecho que, en El Salvador, el término paliativo resulte ser relativamente nuevo dentro del mayor ente de la materia, el Ministerio de Salud (MINSAL) ${ }^{4}$, al punto que, no se cuenta con acreditación oficial para médicos y enfermeras que se dedican a brindar tratamientos y cuidados paliativos y estos son regidos específicamente por las distintas juntas encargadas de la regulación de estas profesiones. Cabe destacar que un equipo para brindar cuidados paliativos debe estar integrado de forma multidisciplinaria por distintos profesionales de la rama de la salud entre lo que se pueden mencionar: médicos, enfermeras, psicólogos, fisioterapeutas, voluntarios, entre otros. Los profesionales de enfermería por ser quienes más acompañamiento brinda al usuario y familia en todas las etapas de la enfermedad necesitan de bases teóricas especializadas que deben ser incorporadas desde su formación académica.

En el país, se inauguraron, a finales de 2013, seis unidades de Cuidados Paliativos y Dolor en los hospitales de segundo y tercer nivel, pero el único centro dedicado a la atención especializada de estos pacientes es el Hospital La Divina Providencia ${ }^{5}$ (PALIAMED, 2018). Aunque el MINSAL ha diseñado programas de atención a enfermos avanzados oncológicos y no oncológicos, que contemplan entre sus servicios los cuidados paliativos, en general, no se cuenta con infraestructura para la atención de los enfermos avanzados, por lo que se dificulta el acompañamiento de la familia en estos espacios, no existe dentro del presupuesto anual del MINSAL un rubro destinado a cuidados paliativos, por lo tanto deben ser destinados del presupuesto general aprobado para cada hospital que cuenta con estas unidades.

En este sentido, la apuesta del MINSAL se ve reflejada en los Lineamientos técnicos para la atención del dolor crónico y cuidados

\footnotetext{
4. Dentro del documento, las autoras se referirán al Ministerio de Salud mediante su acrónimo, MINSAL.

5. Hospital ubicado en la colonia Miramonte de San Salvador, El Salvador.
} 
paliativos $^{6}$, además de mantener los convenios vigentes con el Hospital Divina Providencia y una relación estrecha con los únicos centros privados enfocados en brindar este tipo de cuidados.

Es preciso resaltar el hecho que, al referirse a cuidados paliativos, se busca enfatizar en procedimientos médicos, cuidados de enfermería y atención integral que, si bien ayudan a hacer confortable el proceso de los últimos momentos de vida de las personas, este proceso es totalmente opuesto a la eutanasia y se apoya en todo momento en el derecho que tienen todas las personas al trato digno y a una vida de calidad en todo momento. Los cuidados paliativos constituyen un abordaje de cuidado especial, destinado a mejorar la calidad de vida del paciente con enfermedad avanzada, sin posibilidades de cura, y de sus familiares, por medio de la evaluación y del tratamiento adecuado para el alivio del dolor y de los síntomas, se trata de un soporte psicosocial y espiritual. "Enfermería es una de las profesiones que más contribuye a poder brindar este tipo de cuidados, porque permanece junto al paciente en todas las etapas de la vida" (López, 2013, p.16).

Distintos autores, como Centeno (2018), estiman que las necesidades no cubiertas de cuidados paliativos a nivel mundial afectan a 27 millones de personas cada año; esto debido posiblemente a la falta de personal capacitado para brindar cui- dados con las características que conlleva acompañamiento, entrega, empatía y alivio, todos estos elementos son importantes para mantener el carácter holístico y humanístico de los cuidados que brinda enfermería.

La etapa terminal y la muerte de las personas es algo inevitable, es un momento personal y único, por lo tanto, el papel del personal de salud y específicamente de enfermería, no es buscar la curación, sino, ayudar al bien morir brindando acompañamiento y tranquilidad; sin embargo, los escenarios de atención son diversos y en muchas ocasiones estos cuidados no son recibidos por diversas razones, aunque el paciente lo necesite; entre estas razones, según el Consejo Internacional de Enfermería, se encuentran:

Sobrecarga laboral permanente aunada a bajas remuneraciones que afectan su salud física y psicología de los trabajadores de la salud; en este sentido el Consejo Internacional de Enfermeras (2014), señala que "estas afecciones se agudizan muchas veces con las malas relaciones interpersonales, los problemas concomitantes a las funciones profesionales, y diversos riesgos del lugar de trabajo por ello, la sobrecarga laboral produce desgaste físico y mental, impidiendo al profesional hacer frente con éxito a las situaciones diarias que se presentan, entre ellos brindar cuidados especiales a los usuarios como cuidados paliativos entre otros" (p.4).

6. Este documento contiene las directrices para la aplicación de los cuidados paliativos en todos los niveles del sistema nacional de salud de El Salvador; de tal forma que constituye la principal herramienta de consulta para la investigación al contener los procedimientos oficiales del MINSAL. 
Durante la formación profesional es necesario que el estudiante cumpla con la aprobación de total de las asignaturas, entre las cuales se encuentran las del área básica de enfermería y las del área especializada de enfermería, en las cuales se desarrolla diferentes contenidos que están relacionados a brindar cuidados de enfermería integrales a usuario, basándose en las características de los grupos poblacionales en estudio y patologías de bajo, mediano y alto riesgo, sin profundizar en el estudio con un enfoque especifico de cuidados paliativos.

Por lo antes mencionado, determinar el nivel de conocimientos sobre cuidados paliativos que poseen los estudiantes de $5^{\circ}$ año de Licenciatura en Enfermería de la Universidad Católica de El Salvador, Centro Regional de Ilobasco, resulta importante a fin de justificar una reforma a la malla curricular del nivel académico de licenciatura que permita la formación especializada e integral sobre cuidados paliativos de futuros profesionales.

\section{Metodología}

Se desarrolló una investigación de tipo mixta que buscó conocer la situación de forma general sobre los conocimientos en relación a cuidados paliativos que poseen los estudiantes de la carrera de Licenciatura en Enfermería de la Universidad Católica de El Salvador, Centro Regional de Ilobasco.
Para el ciclo académico I-2020 se contó con una población de 306 estudiantes inscritos en la carrera de Licenciatura en Enfermería, sin embargo, el estudio se ejecutó en el ciclo II-2020 teniendo una población total, en ese momento, de 350 estudiantes inscritos. Así, haciendo uso de la técnica de muestreo no probabilístico de tipo discrecional, se seleccionó el grupo de 42 estudiantes de quinto año la carrera Licenciatura en Enfermería; este grupo resultó de interés para el estudio porque son los estudiantes que egresaron ese año y se integraron a la población productiva que prestará sus servicios ad honoren por seis meses en el MINSAL.

Para recolectar la información se utilizó la técnica de encuesta, con preguntas cerradas, dirigida a los estudiantes de la carrera de Licenciatura en Enfermería sobre las variables conocimiento y cuidados paliativos. El instrumento que se utilizó para recolectar los datos de la investigación fue el cuestionario, donde se identificó entre otros aspectos: datos generales del estudiante, interés por el tema de cuidados paliativos, y el conocimiento que tiene sobre el tema en cuestión, con un total de 25 preguntas que buscaron dar respuesta a los objetivos del estudio.

Además, se realizó entrevista al Dr. Mario López Saca, director de Hospice La Cima? quién es pionero en el país en la implementación y promoción de la aplicación cuidados

7. Centro de medicina paliativa con atención a pacientes mayores de 18 años con patologías como el cáncer y otras enfermedades crónicas avanzadas, ubicado en La Cima III en San Salvador. 
paliativos para médicos, enfermeras y otros miembros del equipo de salud, la entrevista fue mediante la plataforma digital Google Meet y trató sobre generalidades de cuidados y el punto de vista profesional sobre cuidados paliativos y la atención que brindan los profesionales de salud, obteniendo datos cualitativos que fueron presentados para sustentar la investigación.

A través del método analítico se posibilitó retomar datos existentes para iniciar el estudio y completando las siguientes fases en las cuales se reunió información necesaria para establecer la relación a la unidad de análisis retomando cada uno de los indicadores que le dieron origen al instrumento de recolección de datos.

Una vez recolectada, la información fue procesada en forma manual aplicándose el método estadístico porcentual que sirvió para cuantificar los resultados obtenidos y que se presentan al final de la investigación. Se procedió a realizar el análisis basado en los objetivos del estudio resaltando los datos de mayor relevancia y que dieron respuesta a las interrogantes planteadas en el inicio de la investigación.

\section{Resultados}

Se presentan a continuación los resultados más relevantes respecto de las unidades en estudio: conocimiento y cuidados paliativos.

Según los datos obtenidos el $87 \%$ de la población manifestó tener conocimientos de cuidados paliativos, un $13 \%$ respondió que conoce poco sobre los mismos.
Los anteriores resultados son consecuencia de que el pensum académico de los estudiantes de Licenciatura en Enfermería, en la UNICAES, adscribe el estudio de la asignatura Farmacología Aplicada a Enfermería, en la cual se integra la clasificación de los medicamentos, entre ellos los analgésicos. Así, tomando de referencia a la OMS (2015) se estudian los analgésicos subdivididos como no opioides, opioides menores y opioides potentes, los cuales son medicamentos utilizados para el manejo del dolor y la sedación paliativa.

Según los datos obtenidos, el 53\% manifestó conocer el uso de medicamentos para el dolor, $42 \%$ respondió que no conoce su uso y el $5 \%$ expresó que conoce poco sobre el uso de medicamentos para el dolor en pacientes con cuidados paliativos. Es preciso no omitir que existen aseveraciones relacionadas al uso de los opioides para el tratamiento del dolor, se ha podido establecer que el riesgo a desarrollar una adicción a opioides aumenta cuando hay antecedentes de abuso y dependencia a sustancias, presencia de psicomorbilidades, un ambiente social favorecedor al consumo, y cuando se prescriben dosis superiores a $120 \mathrm{mg}$ de morfina equivalente al día (Acuña, 2019).

Además, algunas de las situaciones del conocimiento de los usuarios y que en ocasiones limita la aceptación del uso de opioides para tratamiento del dolor son su administración es señal de muerte inminente y que pueden generar depresión respiratoria, entre otros. Ahora bien, al 
contrastar esta información con una pregunta específica sobre los mitos que existen en relación al uso de opioides, la respuesta fue que el $73.3 \%$ no conoce mitos relacionados a su uso en pacientes con cuidados paliativos.

Sin embargo, desde hace años, el uso de opioides en enfermedades crónicas y en procesos finales de vida ha sido considerado un tema de mucho debate por un gran número de personas, sea o no en el ámbito sanitario. Estas personas tienen cierto recelo respecto al uso de estos fármacos, que en muchas ocasiones podrían beneficiar el mejor control sintomático y de bienestar en diversas etapas de la vida (Villavicencio, 2017). Pero, la realidad es que son fármacos de uso indispensable para el manejo del dolor severo producido por enfermedades en etapa terminal.

Al consultar si en las prácticas en los centros de salud los estudiantes necesitaron brindar cuidados paliativos, el 58\% de la población respondió que no se asignó paciente a quienes fuera necesario brindar cuidados paliativos durante sus prácticas clínicas, un 29\% manifestó que sí, mientras que un $13 \%$ indicó que fueron pocas ocasiones en las que se le asignó paciente con cuidados paliativos.

No en todos los hospitales de segundo nivel asignados a la institución para realizar prácticas existen unidades del dolor, por lo tanto, las oportunidades para que el estudiante aplique este tipo de cuidados se ve limitada, sin embargo, el porcentaje de estudiantes que respondió que tuvo la oportunidad durante las prácticas clínicas que se le asignaran usuarios que necesitaban cuidados paliativos es significativo.

El pensum académico actual de la carrera de Licenciatura en Enfermería, no integra asignatura, ni contenido que incluyan el componente de cuidados paliativos específico, sin embargo, de manera general se abordan cuidados de enfermería a pacientes con enfermedades de bajo, mediano y alto riesgo con mayor énfasis en el componente humanístico; apoyo espiritual, empatía y apoyo a la familia. Para 2022, el nuevo Plan de Estudios introduce la asignatura de Bioética, en la cual se abordan tópicos relacionados a la forma ética de proceder ante las enfermedades terminales, la eutanasia y otros procedimientos médicos de última generación; esta asignatura podría marcar un antes y después en la formación del profesional de la salud de cara a los nuevos retos en el proceder ético de la sociedad moderna.

Por otro lado, es innegable que el conocimiento es parte fundamental de los cuidados de enfermería; tal es la importancia que, haciendo alusión a los inicios de la profesión, Florence Nightingale le brindó el nombre de cuidados profesionales lo que implica la formación y el conocimiento; si bien es cierto que los cuidados paliativos son un término relativamente nuevo implica la aplicación de base científica para poder realizarlo.

Según los datos obtenidos al consultar sobre la orientación recibida sobre cuidados paliativos, el $47 \%$ manifestó haber recibido poca orien- 
tación, mientras que el $45 \%$ respondió que se recibió orientación y un $8 \%$ indicó no haber recibido ninguna orientación sobre cuidados paliativos. Este último dato se vuelve significativo porque el $8 \%$ de los futuros profesionales no conocen de cuidados paliativos.

En cuanto a la consulta sobre la importancia de incluir una asignatura relacionada a cuidados paliativos, Finalmente, el $97 \%$ de la población estudiada respondió que sí es importante incluir en el programa de estudio una asignatura de ese tipo.

Por otro lado, la totalidad de la población respondió estar interesada en participar en cursos o diplomados sobre manejo de dolor crónico y cuidados paliativos.

\section{Discusión}

Sobre la base de los datos recolectados, el rango porcentual de respuesta fue del 100\%, no hubo preguntas sin contestar. La importancia relativa de cada uno de los apartados o ítems determinó los resultados de la aplicación del modelo estadístico que permite extraer conclusiones basadas en hechos medibles, más allá de la interpretación subjetiva. Los resultados apuntaron a que específicamente, respecto de la carrera de enfermería existen dos aspectos determinantes, la necesidad de capacitación y formación de personal con sentido humano.

La diferencia estará en que el personal de salud humanísticamente formado que atiende a enfermos terminales tiene ese componente extra tan valioso en cuidados paliativos, porque de esta manera, al estudiante se le ha instruido para ser compasivo y a la vez a tener conocimientos para apoyar en los momentos del final de la vida. Así mismo, tener empatía de escuchar, comunicar y manejar un lenguaje verbal y no verbal que genere calidez en los momentos angustiosos que sobrevienen con el padecimiento de enfermedades mortales; todo ello, sin dejar desvalorizar la importancia de conocimiento científico para el uso correcto de opioides, administrar analgésicos por vía subcutánea y brindar acompañamiento espiritual.

Finalmente, respecto de la investigación, no puede obviarse el hecho que es difícil opinar sobre la calidad de los propios conocimientos, sin práctica ni experiencia en el área; así como establecer un diagnóstico fundamentado y razonablemente crítico de ello, pero la realización de trabajos como este puede abrir un debate fructífero sobre la necesidad de incorporar los cuidados paliativos en la malla curricular de enfermería o justificar cursos y diplomados en esta área. La herramienta utilizada, además de aportar información para la evaluación de la calidad de un informe de investigación, puede emplearse para dirigir correctamente otros trabajos de investigación con fines diagnósticos, aunque no necesariamente en el último año de la carrera en Licenciatura en Enfermería. 


\section{Referencias}

Asociación Latinoamericana de Cuidados Paliativos. (2012). Atlas de Cuidados Paliativos en Latinoamérica. IAHPC Press. http:/cuidadospaliativos.org/uploads/2012/10/atlas/Atlas\%20 de\%20Cuidados\%20Paliativos\%20en\%20Latinoamerica.pdf

Centeno, C. (2018, 28 de febrero). Todas las vidas son valiosas para la medicina paliativa. Vatican News. https:/www.vaticannews.va/es/mundo/news/2018-02/medicina-paliativa-cuidados-paliativos-doctor-centeno-congreso-.html

Consejo Internacional de Enfermería. (2014). Entornos de Practica Favorables: Lugares de Trabajo de Calidad-Atención de Calidad al Paciente. http://www.icn.ch/images/stories/documents/publications/ind/indkit2007sp.pdf

López, A. (2013). Manual de medicina paliativa. Ediciones Universidad de Navarra.

Ministerio de Salud. (2014). Lineamientos Técnicos para la Atención del Dolor Crónico y Cuidados Paliativos. Viceministerio de Políticas de Salud. http://asp.salud.gob.sv/regulacion/pdf/ lineamientos/lineamientos_tecnicos_atencion_dolor_cronico_y_cuidados_paliativos.pdf

Organización Mundial de la Salud. (2015). Uso de Opioides en el tratamiento del dolor, Manual para Latinoamérica. https://cuidadospaliativos.org/uploads/2012/11/ManualOpioides.pdf

Organización Mundial de la Salud. (2018). Cuidados paliativos. https://www.who.int/es/newsroom/fact-sheets/detail/palliative-care

PALIAMED. (2018). Cuidados Paliativos. https://www.paliamed.org

Perera Milian, L. (2016). Cuidados paliativos en pacientes en estado terminal. Medimay, 23 (2). http://revcmhabana.sld.cu/index.php/rcmh/rt/printerFriendly/1014/1423

Quintana, T. (2016, 18 de noviembre). Espiritualidad en Cuidados Paliativos. Bioética desde Asturias. https://www.bioeticadesdeasturias.com/espiritualidad-en-cuidados-paliativos/

Villavicencio, C. (2017, 17 de agosto). Mitos del uso y efectos de los opioides. Top Doctors. https:/www.topdoctors.es/articulos-medicos/mitos-del-uso-y-efectos-de-los-opioides 